DISSERTATIONES PHILOLOGICAE ARGENTORATENSES SELECTAE

EDIDEREN' B. KEIL ET R. REITZENSTEIN.

VOLUMEN XIV, FASCICULUS III.

\title{
EPISTULAE AMATORIAE QUOMODO COHAEREANT CUM ELEGIIS ALEXANDRLISS.
}

SCRIPSIT

MAXIMILIANOS HEINEMANN.

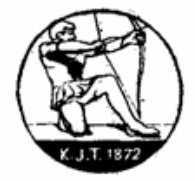

ARGENTORATI

APUD CAROLUM I. TRUEBNER

MCMX. 
M. DuMont Schauberg, Straßburg i. E. 


\section{RICARDO REITZENSTEIN}

\section{BRUNONI KEIL}

PIE'TATIS

ERGO. 
